International Journal of Pure and Applied Mathematics

Volume 114 No. 4 2017, 791-795

ISSN: 1311-8080 (printed version); ISSN: 1314-3395 (on-line version)

url: http://www.ijpam.eu

doi: 10.12732/ijpam.v114i4.9

\title{
SUPERCYCLICITY OF TUPLES IN SOME TOPOLOGIES
}

\author{
B. Yousefi ${ }^{1 \S}$, A. Sharifi ${ }^{2}$ \\ ${ }^{1,2}$ Department of Mathematics \\ Payame Noor University \\ P.O. Box 19395-4697, Tehran, IRAN
}

\begin{abstract}
In this paper, we prove the supercylicity for a tuple of operators in the strong operator topology and in the norm of Hilbert-Schmidt operators. Also, the same result for hypercyclicity of tuples is considered.
\end{abstract}

AMS Subject Classification: 47B37, 47B33

Key Words: strong operator topology, Hilbert-Schmidt operator, tuple of operators, supercyclic vector, supercyclicity criterion, hypercyclic operator, topologically transitive

\section{Introduction}

Let $\mathcal{T}=\left(T_{1}, T_{2}, \ldots, T_{n}\right)$ be an n-tuple of commuting operators acting on an infinite dimensional Banach space $X$. We will let $\mathcal{F}=\left\{T_{1}{ }^{k_{1}} T_{2}{ }^{k_{2}}, \ldots, T_{n}{ }^{k_{n}}: k_{i} \in\right.$ $\left.\mathbf{Z}_{+}, \quad i=1, \ldots, n\right\}$ be the semigroup generated by $\mathcal{T}$. For $x \in X$, the orbit of $x$ under the tuple $\mathcal{T}$ is the set $\operatorname{Orb}(\mathcal{T}, x)=\{S x: S \in \mathcal{F}\}$. A vector $x$ is called a supercyclic (hypercyclic) vector for $\mathcal{T}$ if $\operatorname{COrb}(\mathcal{T}, x)(\operatorname{Orb}(\mathcal{T}, x))$ is dense in $X$ and in this case the tuple $\mathcal{T}$ is called supercyclic (hypercyclic). By $\mathcal{T}_{d}^{(k)}$ we will refer to the set of all $k$ copies of an element of $\mathcal{F}$, i.e. $\mathcal{T}_{d}^{(k)}=\left\{S_{1} \oplus \ldots \oplus S_{k}\right.$ : $\left.S_{1}=\ldots=S_{k} \in \mathcal{F}\right\}$. We say that $\mathcal{T}_{d}^{(k)}$ is hypercyclic provided there exist $x_{1}, \ldots, x_{k} \in X$ such that $\left\{W\left(x_{1} \oplus \ldots \oplus x_{k}\right): W \in \mathcal{T}_{d}^{(k)}\right\}$ is dense in the $k$ copies

Received: December 25, 2016

Revised: $\quad$ February 7, 2017

Published: $\quad$ June 7,2017

(c) 2017 Academic Publications, Ltd. url: www.acadpubl.eu

$\S_{\text {Correspondence author }}$ 
of $X, X \oplus \ldots \oplus X$, and similarly we say that $\mathcal{T}_{d}^{(k)}$ is supercyclic provided there exist $x_{1}, \ldots, x_{k} \in X$ such that $\mathbf{C}\left\{W\left(x_{1} \oplus \ldots \oplus x_{k}\right): W \in \mathcal{T}_{d}^{(k)}\right\}$ is dense in the $k$ copies of $X$. We say that a tuple $\mathcal{T}=\left(T_{1}, T_{2}, \ldots, T_{n}\right)$ is topologically transitive with respect to a tuple of nonnegative integer sequences

$$
\left(\left\{k_{j(1)}\right\}_{j},\left\{k_{j(2)}\right\}_{j}, \ldots,\left\{k_{j(n)}\right\}_{j}\right),
$$

if for every nonempty open subsets $U, V$ of $X$ there exists $j_{0} \in \mathbb{N}$ such that $T_{1}^{k_{j_{0}(1)}} T_{2}^{k_{j_{0}(2)}} \ldots T_{n}^{k_{j_{0}(n)}}(U) \cap V \neq \varnothing$. Also, we say that an n-tuple $\mathcal{T}$ is topologically transitive if it is topologically transitive with respect an n-tuple of nonnegative integer sequences. If $X$ is separable, topologically transitivity of $\mathcal{T}$ implies the hypercyclicity of $\mathcal{T}$. For several works see [1-3].

We begin with the following well known theorems that are the main tools we use to show that a tuple of operators is supercyclic.

Theorem 1.1. Let $X$ be a separable infinite dimensional Banach space and $\mathcal{T}=\left(T_{1}, T_{2}, \ldots, T_{n}\right)$ be a tuple of operators $T_{1}, T_{2}, \ldots, T_{n}$. Then $\mathcal{T}$ is supercyclic, if and only if for any two non-void open sets $U$ and $V$, there exist $m_{i} \geq 1$ for $i=1, \ldots, n$ and and $\lambda \in \mathbb{C} \backslash\{0\}$ such that $\lambda T_{1}^{m_{1}} \ldots T_{n}^{m_{n}}(U) \cap V \neq \varnothing$.

Theorem 1.2. (Supercyclicity Criterion for Tuples of Operators) Let $\mathcal{T}=\left(T_{1}, T_{2}, \ldots, T_{n}\right)$ be an n-tuple of operators acting on an infinite dimensional Banach space $X$. If there exist two dense subsets $Y$ and $Z$ in $X$, and strictly increasing sequences $\left\{m_{j_{i}}\right\}_{j}$ for $i=1, \ldots, n$, and a sequence of mappings $S_{j}: Z \rightarrow X$ such that:

1) $T_{1}^{m_{j_{1}}} T_{2}^{m_{j_{2}}} \ldots T_{n}^{m_{j_{n}}} S_{j} z \rightarrow z$ for every $z \in Z$,

2) $\left\|T_{1}^{m_{j_{1}}} T_{2}^{m_{j_{2}}} \ldots T_{n}^{m_{j_{n}}} y\right\|\left\|S_{j} z\right\| \rightarrow 0$ for every $y \in Y$ and every $z \in Z$, then $\mathcal{T}$ is supercyclic.

\section{Main Results}

We will investigate the supercylicity for a tuple of operators by the three open sets.

Recall that if $\left\{e_{i}\right\}$ is a basis for a separable Hilbert space $H, A \in B(H)$ and $\|A\|_{2}=\left[\sum_{i=1}^{\infty}\left\|A e_{i}\right\|^{2}\right]^{\frac{1}{2}}<\infty$, then $\mathrm{A}$ is called a Hilbert-Schmidt operator. The set of Hilbert-Schmidt operators on $H$ is denoted by $B_{2}(H)$.

Note that $B(H)$ and $B_{2}(H)$ respectively with strong operator topology and \|\|$_{2}$-topology are separable. For this suppose that $\left\{e_{i}\right\}$ is the basis for the separable Hilbert space $H$, also suppose that $S$ is a dense subset in $H$. Then 
$S(H)$ will denote the set of all finite rank operators $E$ such that there exists $N \in \mathbb{N}$ satisfying $E\left(e_{n}\right)=0$ for $n \geq N$ and $E\left(e_{n}\right) \in S$ for $n<N$. Clearly, $S(H)$ is \|\|$_{2^{-}}$-dense in $B_{2}(H)$ and if $S$ is countable, then $S(H)$ is also countable.

For any operator $T \in B(H)$, the left multiplication operator $L_{T}: B(H) \rightarrow$ $B(H)$ is defined by $L_{T}(S)=T S$ for all $S$ in $B(H)$. If $\mathcal{T}=\left(T_{1}, T_{2}, \ldots, T_{n}\right)$ is an n-tuple of operators acting on $H$, then by $\mathcal{L}_{T_{1}, \ldots, T_{n}}$ we mean the tuple $\left(L_{T_{1}}, L_{T_{2}}, \ldots, L_{T_{n}}\right)$.

Lemma 2.1. Let $\mathcal{T}=\left(T_{1}, T_{2}, \ldots, T_{n}\right)$ be an n-tuple of operators in $B(H)$. Then $\mathcal{T}_{d}^{\infty}$ acting on $\bigoplus_{n=1}^{\infty} H$ is unitary equivalent to $\mathcal{L}_{T_{1}, \ldots, T_{n}}$ acting on $B_{2}(H)$.

Proof. Let $\left\{h_{i}\right\}$ be a sequence in $H$, such that $\sum_{i=1}^{\infty}\left\|h_{i}\right\|^{2}<\infty$. Put $h=$ $\bigoplus_{i=1}^{\infty} h_{i}$ and define $U_{h}$ by $U_{h}(x)=\sum_{i=1}^{\infty}<x, e_{i}>h_{i}$ for every $x \in H$. Now consider the linear operator $R: \bigoplus_{n=1}^{\infty} H \rightarrow B_{2}(H)$ defined by $R(h)=U_{h}$. Note that for every $h=\bigoplus_{n=1}^{\infty} h_{i}$ in $\bigoplus_{n=1}^{\infty} H$ we get $\left\|U_{h}\right\|_{2}^{2}=\sum_{i=1}^{\infty}\left\|U_{h} e_{i}\right\|^{2}=$ $\sum_{i=1}^{\infty}\left\|h_{i}\right\|^{2}<\infty$. This implies that $R$ is an isometry. Now we show that $R$ is also surjective. For this suppose that $A$ is an arbitrary Hilbert-Schmidt operator and put $h_{i}=A e_{i}$ for any $i \geq 1$. Then $\sum_{i=1}^{\infty}\left\|h_{i}\right\|^{2}=\sum_{i=1}^{\infty}\left\|A e_{i}\right\|^{2}=\|A\|_{2}^{2}<\infty$. Thus $h=\bigoplus_{i=1}^{\infty} h_{i} \in \bigoplus_{n=1}^{\infty} H$ and $R(h)=A$. Now clearly we can see that $R^{-1} L_{T_{1}}^{k_{1}} L_{T_{2}}^{k_{2}} \ldots L_{T_{n}}^{k_{n}} R=\bigoplus_{i=1}^{\infty} T_{1}{ }^{k_{1}} T_{2}{ }^{k_{2}} \ldots T_{n}{ }^{k_{n}}$ and so the proof is complete.

Theorem 2.2. Let $\mathcal{T}=\left(T_{1}, T_{2}, \ldots, T_{n}\right)$ be an n-tuple of operators acting on $H$. Then $\mathcal{T}$ satisfies the Supercyclicity Criterion if and only if $\mathcal{L}_{T_{1}, \ldots, T_{n}}$ is SOT-supercyclic on $B(H)$.

Proof. Note that $\mathcal{L}_{T_{1}, \ldots, T_{n}}$ is SOT-supercyclic on $B(H)$ if and only if $\mathcal{L}_{T_{1}, \ldots, T_{n}}$ is supercyclic on $B_{2}(H)$ with \|\|$_{2}$-topology. Now Lemma 2.16 implies that $\mathcal{L}_{T_{1}, \ldots, T_{n}}$ is supercyclic on $B_{2}(H)$ with \|\|$_{2}$-topology if and only if for all integer $k \geq 1, \mathcal{T}_{d}^{(k)}$ is supercyclic on $\bigoplus_{i=1}^{k} H$ and this is true if and only if $\mathcal{T}$ satisfies the Supercyclicity Criterion. This completes the proof.

Corollary 2.3. The tuple $\mathcal{T}=\left(T_{1}, \ldots, T_{n}\right)$ satisfies the Supercyclicity Criterion if and only if for any two nonempty SOT-open sets $U$ and $V$ in $B(H)$, there exists some positive integer $k_{1}, \ldots, k_{n} \geq 1$ and $\lambda \in \mathbf{C}$ such that $\lambda L_{T_{1}}^{k_{1}} L_{T_{2}}^{k_{2}} \ldots L_{T_{n}}^{k_{n}} U \cap V \neq \phi$.

Corollary 2.4. If $\mathcal{T}=\left(T_{1}, \ldots, T_{n}\right)$ satisfies the Supercyclicity Criterion, then $\mathcal{L}_{T_{1}, \ldots, T_{n}}$ has a \|\|$_{2}$-dense $G_{\delta}$ set of supercyclic vectors. 
Proof. Since each supercyclic operator on a complete metric space has a dense $G_{\delta}$ set of supercyclic vectors, and $B_{2}(H)$ is complete with \|\|$_{2}$-topology, it is clear.

Theorem 2.5. Let $\mathcal{T}=\left(T_{1}, T_{2}, \ldots, T_{n}\right)$ be an n-tuple of operators acting on $H$. Then the following are equivalent:

i) $\mathcal{T}$ is supercyclic and for each nonempty open subset $U$ and each neighborhood $W$ of zero, $\lambda T_{1}^{m_{1}} T_{2}{ }^{m_{2}} \ldots T_{n}{ }^{m_{n}} U \cap W \neq \phi$ and $\lambda T_{1}{ }^{-m_{1}} T_{2}{ }^{-m_{2}} \ldots T_{n}{ }^{-m_{n}} U \cap$ $W \neq \phi$ for some integers $m_{1}, m_{2}, \ldots, m_{n} \geq 1$ and $\lambda \in \mathbf{C} \backslash\{0\}$.

ii) For each pair $U, V$ of nonempty open subsets of $H$, and each neighborhood $W$ of zero, $\lambda T_{1}^{k_{1}} T_{2}{ }^{k_{2}} \ldots T_{n}{ }^{k_{n}} U \cap W \neq \phi$ and $\lambda T_{1}^{k_{1}} T_{2}{ }^{k_{2}} \ldots T_{n}{ }^{k_{n}} W \cap V \neq \phi$ for some integers $k_{i} \geq 1, i=1, \ldots, n$, and $\lambda \in \mathbf{C} \backslash\{0\}$.

Proof. (i) implies (ii): Let $U, V$ and $W$ be nonempty open sets in $H$ with $0 \in$ $W$. Since $\mathcal{T}$ is supercyclic, by Theorem 1.1 , we have $U \cap \beta T_{1}{ }^{-m_{1}} T_{2}{ }^{-m_{2}} \ldots T_{n}{ }^{-m_{n}} V$ $\neq \phi$ for some positive integers $m_{1}, m_{2}, \ldots, m_{n}$ and $\beta \in \mathbb{C} \backslash\{0\}$. Let $G$ be a neighborhood of zero that is contained in $W \cap \beta T_{1}{ }^{-m_{1}} T_{2}{ }^{-m_{2}} \ldots T_{n}{ }^{-m_{n}} W$. By condition (ii), there exist some positive integer $k_{1}, k_{2}, \ldots, k_{n}$ and $\lambda \in \mathbf{C} \backslash\{0\}$ such that

$$
\begin{aligned}
& T_{1}^{-k_{1}} T_{2}^{-k_{2}} \ldots T_{n}^{-k_{n}} G \cap \lambda\left(U \cap \beta T_{1}^{-m_{1}} T_{2}^{-m_{2}} \ldots T_{n}^{-m_{n}} V\right) \neq \phi, \\
& \lambda G \cap T_{1}^{-k_{1}} T_{2}^{-k_{2}} \ldots T_{n}^{-k_{n}}\left(U \cap \beta T_{1}^{-m_{1}} T_{2}^{-m_{2}} \ldots T_{n}^{-m_{n}} V\right) \neq \phi .
\end{aligned}
$$

But

$$
T_{1}^{-k_{1}} T_{2}{ }^{-k_{2}} \ldots T_{n}{ }^{-k_{n}} G \cap \lambda\left(U \cap \beta T_{1}^{-m_{1}} T_{2}{ }^{-m_{2}} \ldots T_{n}{ }^{-m_{n}} V\right)
$$

is a subset of $T_{1}^{-k_{1}} T_{2}{ }^{-k_{2}} \ldots T_{n}{ }^{-k_{n}} W \cap \lambda U$, thus $T_{1}^{-k_{1}} T_{2}{ }^{-k_{2}} \ldots T_{n}{ }^{-k_{n}} W \cap \lambda U \neq \phi$. Also $\lambda G \cap T_{1}^{-k_{1}} T_{2}^{-k_{2}} \ldots T_{n}^{-k_{n}}\left(U \cap \beta T_{1}^{-m_{1}} T_{2}{ }^{-m_{2}} \ldots T_{n}{ }^{-m_{n}} V\right)$ is a subset of

$$
\lambda \beta T_{1}^{-m_{1}} T_{2}^{-m_{2}} \ldots T_{n}^{-m_{n}} W \cap \beta T_{1}^{-k_{1}} T_{2}^{-k_{2}} \ldots T_{n}^{-k_{n}}\left(T_{1}^{-m_{1}} T_{2}^{-m_{2}} \ldots T_{n}^{-m_{n}} V\right)
$$

that is equal to $\beta T_{1}^{-m_{1}} T_{2}^{-m_{2}} \ldots T_{n}^{-m_{n}}\left(\lambda W \cap T_{1}^{-k_{1}} T_{2}^{-k_{2}} \ldots T_{n}^{-k_{n}} V\right)$. This implies that $T_{1}^{-k_{1}} T_{2}{ }^{-k_{2}} \ldots T_{n}{ }^{-k_{n}} V \cap \lambda W \neq \phi$ and so (ii) holds. On the otherhand, clearly (ii) implies (i) and this completes the proof.

By the techniques used in the proof of Theorems 2.2 and 2.5, we can prove the following theorem and so we omit the proof.

Theorem 2.6. Let $\mathcal{T}=\left(T_{1}, T_{2}, \ldots, T_{n}\right)$ be an n-tuple of operators acting on $H$. Then $\mathcal{T}$ satisfies the Hypercyclicity Criterion if and only if $\mathcal{L}_{T_{1}, \ldots, T_{n}}$ is SOT-hypercyclic on $B(H)$ if and only $\mathcal{L}_{T_{1}, \ldots, T_{n}}$ is hypercyclic on $B_{2}(H)$ with \|\|$_{2}$-topology. 


\section{References}

[1] N.S. Feldman, Hypercyclic tuples of operators and somewhere dense orbits, J. Math. Appl., 346 (2008), 82-98.

[2] B.F. Madore and R.A. Martinez-Avendano, Subsapce hypercyclicity, Journal of Mathematical Analysis and Applications, 375, No. 2 (2011), 502-511.

[3] B. Yousefi, Hereditarily transitive tuples, Rend. Circ. Mat. Palermo, 2011 (2011), doi: 10.1007/S12215-011-0066-y. 
